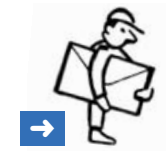

\section{Kostspielige TARMED-Updates}

Mein Mann betreibt eine ländliche Hausarztpraxis. Er ist mit der Behandlung seiner Patienten und dem dazugehörenden administrativen Aufwand ausgelastet. Um so wichtiger ist es, dass ich ihn als Mitarbeiterin (Krankenschwester AKP, dann DN II, aktuell Pflegefachperson) in der Praxis unterstütze, so zum Beispiel mit dem Update zum TARMED 1.05. Die ganze TARMED-Geschichte haben wir längst anzuwenden gelernt. Wir sind richtiggehend Anwenderprofis.

Eigentlich sind wir sehr zufrieden mit unserer Praxiseinrichtung, da wir auf dem neusten Stand sind. Alles funktioniert beim Behandeln, Verrechnen und Abrechnen. Nur eines stört ... die häufigen TARMED-Bereinigungen.

Uns kommen die eigentlich kleinen Änderungen in der Version 1.05 wie Röntgenpositionen von 30.xxxx auf 39.xxxx sehr teuer zu stehen.

Ende März 2007 kostete uns das Update 1459.05 Franken. Nun, am 10. Januar 2008, dürfen wir für diese minimalen Änderungen für den Allgemeinpraktiker wiederum Fr. 1018.95 bezahlen. Wohlgemerkt machen wir alle Updates selber. Diese Beiträge sind reine Lizenzgebühren. Sicherlich, das Softwarehaus hat keine einfache Aufgabe. Die unanständig kurzen Fristen mit x Verschiebedaten über die Feiertage bringen nur Unruhe. Dazu kommt noch die falsche Publikation von Position 39.2100 anstelle 39.2000. Für die SÄZ oder die santésuisse ist es mit einem Korrigendum einfach und rasch korrigiert. Ich hätte länger zu überlegen, wo der Fehler liegt, wenn mir die Softwarefirma nur 39.2000 als Position liefert.
Übrigens sind wir in unserer Arztpraxis ebenfalls in der Lage, einen Verbandswechsel von $10,1 \mathrm{~cm}$ bis $20 \mathrm{~cm}$ oder gar grösser durchzuführen. Dem Allgemeinpraktiker wurde diese Position nicht gewährt.

Die Methadonposition pro Tag ist vor allem zeilenintensiv und papierfüllend. Die Abgaben werden ja in der Regel über drei Monate erfasst. Gegenüber der Lösung vor der Version 1.05 «Pauschale pro Woche» ist dies ein deutlicher Rückschritt.

Myra Wälti, Freidorf TG

\section{Replik}

Sehr geehrte Frau Wälti

Die TARMED-Updates erfolgen jeweils auf das neue Jahr und beinhalten alle Veränderungen in der TARMED-Tarifstruktur, die seit der letzten Version von den Tarifpartnern beschlossen wurden. Je nach Fachgruppe gibt es einmal mehr oder weniger Veränderungen. Mit der Revision des Kapitels 30 (bildgebende Verfahren) konnten für die Radiologen, aber auch für die Grundversorger im Röntgen und für Grundversorger und Spezialärzte im Ultraschallbereich wesentliche Verbesserungen in der Tarifstruktur beschlossen werden. Was die kurzen Fristen betrifft, habe ich in der SÄZ Nr. 4/2008 ausführlich informiert. Solche Feuerwehrübungen sollten in Zukunft nicht mehr nötig sein.

Die Verrechnung der Methadonabgabe entspricht einer Forderung der grundversorgenden Ärzte, die Methadon abgeben, und wurde dementsprechend umgesetzt.

Sie sehen, die Revision der TARMED-Struktur ist sehr wichtig, nur so können Fehler ausgemerzt werden. Die Gewichtung ist jeweils für verschiedene Fachgruppen unterschiedlich.

Dr. med. Ernst Gähler, Mitglied des Zentralvorstands der FMH 